\title{
Placenta's impact factor 2018
}

Yoel Sadovsky, Martin Knöfler, Anthony V. Perkins

As the Editors of Placenta, we are happy to share with our International Federation of Placenta Associations (IFPA) community and all placenta enthusiasts that the 2018 impact factor (IF) of Placenta, released in June 2019, increased from 2.43 to 2.77. When compared to 2017 data, Placenta's 2018 IF advanced from \#16 to \#12 in the ranking of reproductive biology journals, and from \#29 to \#20 among OBGYN journals.

The impact factor for any journal, published each June in the Journal Citation Reports by Clarivate Analytics, is the quotient of all citations of manuscripts published in that journal in the preceding 2 years (this year's IF included $2016+2017$ citations), divided by the number of publications in that journal in the preceding 2 years (again, $2016+2017$ ). When we reviewed the data for 2018, a clear trend emerged: the number of citations increased by $20 \%$, while the number of accepted manuscripts increased by only $5 \%$, which explains the overall IF increase.

Should we be happy? Of course, after the decline of Placenta's IF in the past 3 years, we are happy that the journal impact has risen. Notwithstanding the intense criticism across the scientific world about the validity of the IF as a measure of a journal's quality and importance, the abuse of the IF as a measure of a specific manuscript's impact, and the misuse of the IF by individuals and institutions, the IF is still commonly used to evaluate a journal's quality and "impact", particularly when used to compare to other journals within the same biological discipline. More citations suggest that better manuscripts were published, which implies that better science was performed. 
How can Placenta's impact be further improved? Again, the assumption is that higher quality articles will attract more citations, and more citations of the same number of manuscripts will improve the IF, thus attracting better submissions, which will enable the selection of better manuscripts for publication, leading to more citations, and so on. However, a higher IF that attracts more submissions might pressure the Editors to accept more manuscripts, which may lead to acceptance of more mediocre manuscripts and therefore decrease the number of citations per manuscript and reduce the IF.

What can be ethically done to improve Placenta's quality and consequently, improve the IF? As editors, we can attest that our goal has always been to stimulate the best science and accept the best submissions, not a higher number of submissions. Best submissions are assessed through our peer review process by members of our Editorial Board and all reviewers within our community. Good reviews lead to publication of better science, and bad reviews do the opposite. We have not adopted any policies or dubious strategies designed to improve the IF. We have not engaged in deliberate policies to accept more reviews and have not tried to accept more reviews earlier in the year, so they have more time to be cited during the subsequent academic year. We have not coerced authors to cite manuscripts from Placenta or made this a pre-condition for publication. We have not written editorials that include many citations of Placenta manuscripts in order to artificially inflate our IF. We want to publish important, interesting, novel, creative, relevant, and impactful scientific work that truly propels our field forward. We also encourage new investigators to submit their best work to our journal. While Placenta may not be able to attract "CNS" (Cell, Nature or Science) quality manuscripts (although we would like to), we may remind ourselves that some of the best work that led, for example, to a Nobel Prize, was not published in "CNS". For example, the manuscript that describes the Nobel-winning (1953) discovery of the citric acid cycle by Hans Krebs was rejected by Nature, and ultimately published in Enzymologia. 
The responsibility to improve Placenta's IF should not just fall on the editors' shoulders. The most critical parameters that determine the quality of manuscripts published in Placenta are the quality of submitted manuscripts and the quality of the review process. These responsibilities are shared by all members of our placenta community and should not be taken lightly. We look forward to working with all placenta enthusiasts to deepen our investigation and enhance the impact of our field on scientific knowledge. 